\title{
Evaluation of Surface Enhanced Raman Spectroscopy (SERS) for Detection of Acetone in the Context of Food Safety and Quality Application
}

\author{
Punyatoya Mohapatra \\ Department of Agricultural and Biosystems Engineering \\ North Dakota State University, Fargo, ND 58108, USA \\ Tel: 1-701-367-4904 E-mail:punyatoya@gmail.com \\ Suranjan Panigrahi (Corresponding author) \\ Department of Agricultural and Biosystems Engineering \\ North Dakota State University, Fargo, ND 58108, USA \\ $\&$ \\ Department of Electrical and Computer Engineering Technology \\ Purdue University, Knoy 155, 401 N. Grant Street, West Lafayette, IN 47907, USA \\ Tel: 1-765-494-6908Ｅ-mail: spanigr@purdue.edu

$\begin{array}{ll}\text { Received: November 3, } 2011 & \text { Accepted: November 14, 2011 Published: February 1, } 2012 \\ \text { doi:10.5539/jfr.v1n1p3 } & \text { URL: http://dx.doi.org/10.5539/jfr.v1n1p3 }\end{array}$

The research is financed by USDA-CSREES and conducted at Bioimaging and Sensing Center at North Dakota State University.

\begin{abstract}
Development of sensors for detection of contaminated food products is important to ensure safety of the community. This paper focuses on detection of a compound, acetone associated with pathogen contamination and spoilage of beef in packaged condition. Surface Enhanced Raman Spectroscopy (SERS) technique was used to develop an integrated sensing system. This sensing system utilized laboratory prepared silver sol as the SERS substrate to detect acetone at low ppm and high ppb concentration. A set of eight experiments with two different experimental conditions were conducted. The first four set provided an estimated lower detection limit (LDL) ranging between $69.63 \mathrm{ppm}$ and $630.17 \mathrm{ppm}$ corresponding to the $1711 \mathrm{~cm}^{-1}$ and $532 \mathrm{~cm}^{-1}$ Raman shift $\left(\mathrm{cm}^{-1}\right)$ regions, respectively. The second four sets of experiments provided a lower estimated LDL ranging between 9.78 ppm and $43.45 \mathrm{ppm}$ corresponding to $1222 \mathrm{~cm}^{-1}$ and $532 \mathrm{~cm}^{-1}$, respectively. This study demonstrated the ability of this sensing system to detect the indicator compound.
\end{abstract}

Keywords: Surface enhanced Raman spectroscopy, Lower detection limit, Acetone, Food safety, Beef

\section{Introduction}

Physical and biological activities of pathogenic and spoilage bacteria that cause food safety problems produces metabolites. The metabolites generated in liquid and gaseous form accumulate in the headspace and absorbent sheath of the packaged beef. These metabolites consist of multiple compounds and these compounds can be used as indicators for determination of spoilage and contamination in packaged beef. It is to be noted that many of these compounds are present in trace in parts per million or parts per billion. Hence, detection of these compounds present in parts per million (ppm) or parts per billion (ppb) levels can help consumers detect quality and safety issues associated with packaged beef and reduce the risk of contamination and spoilage in food products. The current sensing techniques are time consuming, tedious, and prone to human error. Therefore, there is a need to develop a robust, reliable, and accurate sensing method to detect indicator compounds present 
inside packaged beef.

This study was a part of a large project where we conducted a systems approach to address these issues from multiple directions. Thus, our larger study focused on developing sensors based on the compounds present in the gaseous and liquid metabolite. Some of our research related to the development of olfactory sensors based on gaseous metabolite are reported in (Balasubramanian et al., 2008), (Balasubramanian, Panigrahi, Logue, Gu, \& Marchello, 2009), and (Amamcharla, Panigrahi, Logue, Marchello, \& Sherwood, 2010). These sensors could constitute an integrated sensing system.

For the development of sensors related to detecting compounds present in liquid metabolites, we postulate that a versatile, portable, low cost SERS based sensor capable of detecting the individual compounds associated with pathogen contamination in beef would be beneficial. The proposed sensing system will be a component of the integrated sensing system consisting of multiple sensing components where each component will respond to different key indicator compounds. A preliminary study from our group indicated acetone as one of the compounds of interests associated with spoilage and pathogen contamination of beef inside the packaged beef (Bhattacharjee et al., 2010). Hence, development of a sensing system to detect acetone at low $\left(10^{2}-10^{3}\right) \mathrm{ppm}$ concentrations could be one of the components of an integrated sensor system. In this study, SERS technique using silver sol as SERS substrate has been used as a sensing system to detect target indicator compound (acetone) in the context of food safety application.

SERS is a rapid and a reliable technique that requires no sample preparation. Several studies have been conducted on identification of different types of liquid analytes for different applications. Silver-doped sol gel films coated on glass substrate exhibited strong enhancement (24000 fold) of Raman scattering of benzoic acid over a period of 48 hours (Y.-H. Lee, Dai, \& Young, 1997). Another application of SERS involved the determination of cyanide in wastewater by low-resolution SERS on a sol-gel substrate. Laboratory prepared gold sol-gel substrate using $785 \mathrm{~nm}$ diode laser provided a limit of detection of $10 \mathrm{ppb}$ (Premasiri, Clarke, Londhe, \& Womble, 2001). Another study conducted by Norrod et al.(1997) compared five SERS substrates in terms of sensitivity and limit of detection. Vapor deposited silver films, electrochemically roughened silver electrodes, nitric acid etched silver foil, Tollens reagent produced silver film and photoreduced silver films on $\mathrm{TiO}_{2}$ ( Titanium oxide) were compared. Out of all of these, post deposition annealed silver films exhibited a limit of detection of $0.36 \pm 0.02$ femtomoles for trans-1, 2-bis (4-pyridyl) ethane (BPE). Another interesting application was the application of SERS for the identification of anthraquinone dyes used in works of art (Chen et al., 2006). Ag- $\mathrm{Al}_{2} \mathrm{O}_{3}$ SERS substrate was prepared by spin coating an alumina nanoparticle layer onto a glass slide followed by deposition of a layer of thermally evaporated silver nanoparticles on top of the alumina layer. This substrate provided an estimated limit of detection of $7 \times 10^{-15} \mathrm{~g}$ for alizarin used as dye in the works of art. Recent applications reported the use of SERS for detection and discrimination of polychlorinated biphenyl (PCB), a commonly known environmental contaminant. Partition layer-modified nanostructured substrates were used to develop reusable SERS sensing mechanism for detection of PCB congeners (Bantz \& Haynes, 2009). Studies showed that it would be possible to detect $50 \mathrm{pM}$ of PCB without any instrument or substrate optimization.

A silver-coated polypropylene filter (AgPPF) was used as a highly sensitive and promising SERS substrate (Bhandari, Walworth, \& Sepaniak, 2009). The study reported the use of SERS for the development of a reproducible SERS substrate for detection of environmentally significant compounds, which includes selected pharmaceuticals, personal care products, and possible endocrine disruptors. In this study, it was found that endocrine disruptors such as Apigenin and Daidzein, were detected down to $5 \times 10^{-8} \mathrm{M}$ and $1 \times 10^{-7} \mathrm{M}$, respectively, without a preconcentration step. An important study conducted by Yaffe \& Blanch (2008) discussed the anomalies in the SERS spectra of biological molecules caused by the use of a wide range of aggregating agents for hydroxylamine-reduced and citrate-reduced silver colloid SERS substrates. The study reported the effect of aggregating agents resulting in anomalous bands in the spectra of analytes. The study recommended the acquisition of SERS spectra of silver colloids and aggregating agent prior to the analyte to observe any peaks due to the silver colloid.

A recent study reported the use of SERS using silver colloidal nanoparticles for detection of azole, widely used as a corrosion inhibitor in copper (Pergolese, Muniz-Miranda, \& Bigotto, 2008). This study compared the interaction of azole with silver colloidal nanoparticles with the normal Raman spectra. It was observed that analyte molecule reacted with the silver nanoparticles producing a shift in the Raman spectrum. This phenomenon was analyzed by density functional theory (DFT) calculations to detect azole.

From the above studies, it was observed that SERS provided fingerprint information about specific chemical compounds. This technique has been widely used for the detection of compounds at different concentrations. 
However, no research has been reported on application of SERS for detection of specific indicator compound associated with pathogen contamination. Therefore, the objective of this study is the evaluation of a low cost Raman spectrometer sensing system using silver sol as SERS substrate for detection of target indicator compound, acetone present in low ppm concentration.

\section{Materials and Methods}

\subsection{Preparation of Silver Sol (SERS substrate)}

Silver sol was synthesized in the laboratory following a standard protocol described by Lee \& Meisel (1982). Deionized water $(100 \mathrm{ml})$ was heated on a stirrer hot plate to $45^{\circ} \mathrm{C} .18 \mathrm{mg}$ of $99 \%$ silver nitrate $\left(\mathrm{AgNO}_{3}\right)$ was added to deionized water and the solution was brought to boiling. Upon further heating, $2 \mathrm{ml}$ of $1 \%$ trisodium citrate was added to the boiling solution. The solution was boiled for another 15 minutes. The resulting chemical reaction produced a greenish-grey colored sol. The silver sol was allowed to cool to room temperature before use. The sol had a maximum absorbance at $420 \mathrm{~nm}$ with an average particle size of $50 \mathrm{~nm}$.

\subsection{Design of Experiment}

Four different concentrations of acetone; 800 ppm, 500 ppm, 200 ppm, and $100 \mathrm{ppm}$ were prepared using the series dilution method. The blank was used as a standard to determine the lower detection limit.

A total of eight experiments, with two different experimental conditions were conducted. Each condition had four sets of experiments. The detailed experimental design is shown in Figure 1. An equal volume of acetone was mixed with silver sol and placed inside a quartz cuvette (Starna Cells Inc., CA, USA). The content of the quartz cuvette was shaken vigorously using a vortex mixer (VWR Scientific, IL, USA) to ensure proper mixing of the two components. Figure 2 shows the integrated Raman system for acquisition of SERS spectra of acetone with a schematic representation of the system alignment. The sample was placed in a cuvette and kept in proper position under the fiber optic probe. The incident light from the laser passed through the fiber optic probe and caused the excitation inside the sample. The excited beam was carried through the fiber optic probe and the raw SERS spectrum was collected. Figure 3 shows a flow chart describing the process to acquire the SERS spectra using the integrated SERS system. SERS intensity of peak height corresponding to eight peak wavenumbers was identified as a suitable feature for determination of LDL. A standard curve of acetone obtained from Enwave Optronics, Inc., CA, USA ("Enwave Optronics, Inc.," n.d.). was used to select peak wavenumbers as a feature for determination of LDL. Table 1 shows the eight different peak wavenumbers selected from the standard acetone curve as promisible feature for estimating LDL. The peak wavenumbers were $532 \mathrm{~cm}^{-1}, 766 \mathrm{~cm}^{-1}, 898$ $\mathrm{cm}^{-1}, 1069 \mathrm{~cm}^{-1}, 1222 \mathrm{~cm}^{-1}, 1353 \mathrm{~cm}^{-1}, 1427 \mathrm{~cm}^{-1}$, and $1711 \mathrm{~cm}^{-1}$ respectively. An estimated lower detection limit (LDL) was determined using peak height corresponding to the peak wavenumber as the feature. The detailed logic to estimate the LDL is described below:

The detailed logic to estimate the LDL is described below:

(1) The raw SERS spectrum (R) was collected for a wavenumber region $\lambda^{-1}\left(0-1976 \mathrm{~cm}^{-1}\right)$ for all the samples using Spectra Suite Software provided by (Ocean Optics Inc, USA).

(2) The raw spectrum (R) was smoothed by 5-point, Savitzky- Golay algorithm using GRAMS-32 software. The smoothed spectrum (S) was saved for further processing.

(3) A zero offset was performed on the smoothed spectra (S) using the in-house developed Macro.

(4) Then offset spectrum $(O)$ was divided into eight peak wavenumbers $\left(\lambda^{-1}{ }_{i=1-8}\right)$ based on standard acetone curve described in the above paragraph. A range of $\pm 10 \mathrm{~cm}^{-1}$ was used to calculate the peak height $(\mathrm{h})$ corresponding to the peak wavenumber $\left(\lambda^{-1}\right)$.

(5) The peak height (h) corresponding to the peak wavenumber was used as a feature to calculate the estimated LDL.

Out of the several analytical figures of merit, lower detection limit, (LDL) is one of the most commonly used parameters to interpret the minimum detectable concentration. Various researches have reported the use of LDL to quantify the amount of analyte. Silver nanoparticles were used for detection of folic acid in water and human serum by Stokes et al. (2008). A LDL of $0.018 \mu$ molar was reported at $514.5 \mathrm{~nm}$. The LDL could be further lowered to $0.001 \mu$ molar by increasing the accumulation time of the sample. A study conducted by Tripp et al. (2008) reported the limit of detection of virus ranging between 1-10 pfu by considering peak area under the curve for a stretching band of a functional group. Bao et al. (2003) have reported the use of silver-doped sol-gel film as a Surface- Enhanced Raman substrate for detection of uranyl and neptunyl ions. The calibration curve provided a detection limit of $8.5 \times 10^{-8} \mathrm{M}$. Silver colloid in sol-gel derived xerogel was used for analysis of phenylacetylene and biphenyl. The substrate provided a lower detection limit of $9 \mathrm{ppb}$ and $135 \mathrm{ppb}$ for phenylacetylene and biphenyl respectively (Lucht, Murphy, Schmidt, \& Kronfeldt, 2000). From the above 
information, it can be observed that lower detection limit (LDL) is one of the commonly used analytical figures of merit to quantify a given concentration of the chemical. Hence, in the current work, LDL has been selected to determine the lowest concentration of acetone in a detectable region.

As per the qualitative definition, LDL refers to minimum concentration or mass of analyte that can be detected at a known confidence level. This limit depends upon the ratio of the magnitude of the analytical signal to the size of the statistical fluctuations in the blank signal (Long \& Winefordner, 1983).The lower detection limit (LDL) was calculated using the following equation:

$$
L D L=\frac{3 \sigma b}{m}
$$

where, $\mathrm{LDL}=$ lower detection limit, $\sigma \mathrm{b}=$ Standard deviation of blank, $\mathrm{m}=$ slope of the curve

\section{Results and Discussion}

This study focused on detection of acetone at different concentration using silver sol as the SERS substrate. Silver sol produced an approximate enhancement factor of 56 . The normal Raman spectrum of acetone showed a lower peak intensity of 750 intensity units as compared to higher peak intensity of $42 \times 10^{3}$ intensity units corresponding to the same wavenumber of $786 \mathrm{~cm}^{-1}$ (Figure 4) using silver sol as the SERS substrate. It was evident that SERS spectra of acetone showed more pronounced peaks as compared to the normal Raman spectrum of acetone.

\subsection{Determination of estimated LDL for condition-1}

As described in the flow chart provided in Figure 1, in condition-1, three replications were obtained on three separate quartz cuvettes. A sample plot of estimated LDL for condition-1 has been provided in Figure 5. It can be observed that a correlation coefficient of 0.91 exists between the SERS intensity and acetone concentration in, $\mathrm{ppm}$. There is variability amongst the replications for a given concentration of acetone. This is explained by the use of three separate cuvettes for three different replications. The position of the cuvette below the fibre optic probe could also vary from one replication to another resulting in higher variation amongst the replication.

It can be observed from Table 2 that Expt-1, 2, and 4 provided lowest estimated LDL of $254.11 \mathrm{ppm}, 198.04 \mathrm{ppm}$, and $69.63 \mathrm{ppm}$ respectively corresponding to peak wavenumber of $1711 \mathrm{~cm}^{-1}$. However, Expt-3 provided the lowest estimated LDL of $189.25 \mathrm{ppm}$ corresponding to $1069 \mathrm{~cm}^{-1}$ peak wavenumber. The correlation coefficients ranging between $0.55-0.90$ were obtained across the four experiments performed under condition- 1 .

It was observed that $532 \mathrm{~cm}^{-1}$ wavenumber of the spectrum provided the highest estimated LDL of $630.17 \mathrm{ppm}$ and $1711 \mathrm{~cm}^{-1}$ wavenumber provided the lowest estimated LDL of $69.63 \mathrm{ppm}$ for condition-1.

\subsection{Determination of estimated LDL for condition-2}

As described in the flow chart provided in Figure 1, in condition-2, three replications were obtained on same quartz cuvettes. Estimated LDL was calculated for individual experiments corresponding to eight peak wavenumbers. Table 3 shows the estimated LDL for four different experiments conducted under condition-2. It can be observed that Expt 1, 2, and 4, provided the lowest estimated LDL of $29.05 \mathrm{ppm}, 27.95 \mathrm{ppm}$, and 17.9 ppm respectively corresponding to peak wavennumber of $898 \mathrm{~cm}^{-1}$. Figure 6 shows the lowest estimated LDL of $69.63 \mathrm{ppm}$ obtained in condition-1 at $1711 \mathrm{~cm}^{-1}$ as compred to $9.78 \mathrm{ppm}$ LDL obtained in condition-2 at $1222 \mathrm{~cm}^{-1}$. Since, condition-2 had less variability amongst the replications; it resulted in a higher correlation coefficient ranging between $0.92-0.96$ across the four different experiments.

It can be observed that particular wavenumber $\left(532 \mathrm{~cm}^{-1}\right)$ of the entire spectral region provided the highest estimated LDL and another specific region $\left(898 \mathrm{~cm}^{-1}\right)$ provided the lowest estimated LDL for condition-2.

\subsection{Comparative study of condition-1 and condition-2 and implications on real world applications}

A study was performed between the two experimental conditions to observe the variations of estimated LDL. It was observed that for a given peak wavenumber, $532 \mathrm{~cm}^{-1}$; condition- 1 provided a higher estimated LDL of $630.17 \mathrm{ppm}$ as compared to $43.45 \mathrm{ppm}$ for condition-2. Comparison of data obtained from Table 2 and 3 shows that; condition-1 provided higher estimated LDL for all peak wavenumbers than condition-2.There are several possible reasons resulting in the higher variation in estimated LDL. One of the major reasons is the high sensitivity of the Surface Enhanced Raman spectroscopy technique. The reason for lack of reproducibility can be divided into two components; (a) instrument component and (b) substrate component.

(a) The instrument component consisted of the alignment of the sample, optimum focal length between the 
fiber optic probe and the sample, and the lighting conditions. The cuvette was placed at an angle of $45^{\circ}$ with respect to the fiber optic probe to acquire the spectra. This angle was measured manually during each replication. The slightest displacement in the cuvette position can result in displacing the optimum focal length resulting in lack of reproducibility of the spectra. Proper lighting condition could be another factor responsible for widely scattered observations between the replications. Diffused light from other sources could interfere with the performance of the system resulting in poor reproducible spectra.

(b) The substrate component consisted of the nature and property of the SERS substrate. In this study, silver sol was used as the SERS substrate. Silver sol was prepared in a batch process. Hence, each experiment was conducted with a different batch of SERS substrate. This could have resulted in lack of reproducibility from sample to sample.

\section{Summary and Conclusions}

The present study was conducted to detect an indicator compound (acetone) associated with contamination and spoilage inside the packaged beef. Surface Enhanced Raman spectroscopy was used as a novel sensing technique to detect acetone at different concentrations. Silver sol was used as a SERS substrate to enhance the spectral signature and a customized portable Raman Spectroscopy system was integrated.

This study showed the potential ability of SERS as a sensing technique to detect acetone at different concentrations. A comparative study of two different conditions showed that condition-2 provided a lower estimated LDL of $9.78 \mathrm{ppm}$ than $69.63 \mathrm{ppm}$ for condition-1.The variation within the replications due to separate cuvettes resulted in higher estimated LDL for condition-1.This variability was avoided in condition-2 by using single cuvette to acquire the replications for a given concentration. This study showed the potential of SERS sensing technique to detect acetone to an estimated LDL of $9.78 \mathrm{ppm}$. However, further scope of this study will involve evaluating the performance of other SERS substrates to further lower the LDL. Additional studies are recommended to further validate this finding on a larger data set and in real world packaged beef samples.

\section{References}

Amamcharla, J. K., Panigrahi, S., Logue, C. M., Marchello, M., \& Sherwood, J. S. (2010). Fourier transform infrared spectroscopy (FTIR) as a tool for discriminating Salmonella typhimurium contaminated beef. Sensing and Instrumentation for Food Quality and Safety, 4(1), 1-12. http://dx.doi.org/10.1007/s11694-009-9090-4

Balasubramanian, S., Panigrahi, S., Logue, C., Doetkott, C., Marchello, M., \& Sherwood, J. (2008). Independent component analysis-processed electronic nose data for predicting Salmonella typhimurium populations in contaminated beef. Food Control, 19(3), 236-246. http://dx.doi.org/10.1016/j.foodcont.2007.03.007

Balasubramanian, S., Panigrahi, S., Logue, C., Gu, H., \& Marchello, M. (2009). Neural networks-integrated metal oxide-based artificial olfactory system for meat spoilage identification. Journal of Food Engineering, 91(1), 91-98. http://dx.doi.org/10.1016/j.jfoodeng.2008.08.008

Bantz, K. C., \& Haynes, C. L. (2009). Surface-enhanced Raman scattering detection and discrimination of $\begin{array}{llll}\text { polychlorinated biphenyls. } & \text { Vibrational 29-35. }\end{array}$ http://dx.doi.org/10.1016/j.vibspec.2008.07.006

Bao, L., Mahurin, S. M., Haire, R. G., \& Dai, S. (2003). Silver-Doped Sol-Gel Film as a Surface-Enhanced Raman Scattering Substrate for Detection of Uranyl and Neptunyl Ions. Analytical Chemistry, 75(23), 6614-6620. http://dx.doi.org/10.1021/ac034791+

Bhandari, D., Walworth, M. J., \& Sepaniak, M. J. (2009). Dual Function Surface-Enhanced Raman Active Extractor for the Detection of Environmental Contaminants. Applied Spectroscopy, 63, 571-578. http://dx.doi.org/10.1366/000370209788347002

Bhattacharjee, P., Panigrahi, S., Lin, D., Logue, C. M., Sherwood, J. S., Doetkott, C., \& Marchello, M. (2010). A comparative qualitative study of the profile of volatile organic compounds associated with Salmonella contamination of packaged aged and fresh beef by HS-SPME/GC-MS. Journal of Food Science and Technology, 48, 1-13. http://dx.doi.org/10.1007/s13197-010-0138-6

Chen, K., Leona, M., Vo-Dinh, K. C., Yan, F., Wabuyele, M. B., \& Vo-Dinh, T. (2006). Application of surface-enhanced Raman scattering (SERS) for the identification of anthraquinone dyes used in works of art. Journal of Raman Spectroscopy, 37(4), 520-527. http://dx.doi.org/10.1002/jrs.1426

Enwave Optronics, Inc. (n.d.). . Retrieved November 14, 2008, from http://www.enwaveopt.com/spectra.htm

Lee, P. C., \& Meisel, D. (1982). Adsorption and surface-enhanced Raman of dyes on silver and gold sols. The 
Journal of Physical Chemistry, 86(17), 3391-3395. http://dx.doi.org/10.1021/j100214a025

Lee, Y.-H., Dai, S., \& Young, J. P. (1997). Silver-doped sol-gel films as the substrate for surface-enhanced Raman scattering. Journal of Raman Spectroscopy, 28(8), 635-639. http://dx.doi.org/10.1002/(SICI)1097-4555(199708)28:8\%3C635::AID-JRS152\%3E3.0.CO;2-0

Long, G. L., \& Winefordner, J. D. (1983). Limit of detection. A closer look at the IUPAC definition. Analytical Chemistry, 55(7), 712A-724A. http://dx.doi.org/10.1021/ac00258a001

Lucht, S., Murphy, T., Schmidt, H., \& Kronfeldt, H.-D. (2000). Optimized recipe for sol-gel-based SERS $\begin{array}{lllll}\text { substrates. Journal of Raman } & \text { Spectroscopy, } & 31(11), & \text { 1017-1022. }\end{array}$ http://dx.doi.org/10.1002/1097-4555(200011)31:11\%3C1017::AID-JRS638\%3E3.0.CO;2-V

Norrod, K. L., Sudnik, L. M., Rousell, D., \& Rowlen, K. L. (1997). Quantitative Comparison of Five SERS Substrates: Sensitivity and Limit of Detection. Applied Spectroscopy, 51(7), 994-1001. http://dx.doi.org/10.1366/0003702971941377

Pergolese, B., Muniz-Miranda, M., \& Bigotto, A. (2008). Surface enhanced Raman spectroscopic studies on 1H-1, 2, 4-triazole adsorbed on silver colloidal nanoparticles. Vibrational Spectroscopy, 48(2), 202-205. http://dx.doi.org/10.1016/j.vibspec.2008.02.007

Premasiri, W. R., Clarke, R. H., Londhe, S., \& Womble, M. E. (2001). Determination of cyanide in waste water by low-resolution surface enhanced Raman spectroscopy on sol-gel substrates. Journal of Raman Spectroscopy, 32(11), 919-922. http://dx.doi.org/10.1002/jrs.762

Stokes, R. J., McBride, E., Wilson, C. G., Girkin, J. M., Smith, W. E., \& Graham, D. (2008). Surface-Enhanced Raman Scattering Spectroscopy as a Sensitive and Selective Technique for the Detection of Folic Acid in Water and Human Serum. Applied Spectroscopy, 62(4), 371-376. http://dx.doi.org/10.1366/000370208784046812

Tripp, R. A., Dluhy, R. A., \& Zhao, Y. (2008). Novel nanostructures for SERS biosensing. Nano Today, 3(3-4), 31-37. http://dx.doi.org/10.1016/S1748-0132(08)70042-2

Yaffe, N. R., \& Blanch, E. W. (2008). Effects and anomalies that can occur in SERS spectra of biological molecules when using a wide range of aggregating agents for hydroxylamine-reduced and citrate-reduced silver colloids. Vibrational Spectroscopy, 48(2), 196-201. http://dx.doi.org/10.1016/j.vibspec.2007.12.002

Table 1. Peak wavenumbers of acetone obtained from standard Raman spectral signature. Source: http://www.enwaveopt.com/spectra.htm

\begin{tabular}{|c|c|}
\hline Sl.no. & Peak Wavenumber $\left.\mathbf{( c m}^{-\mathbf{1}}\right)$ \\
\hline 1 & 532 \\
\hline 2 & 766 \\
\hline 3 & 898 \\
\hline 4 & 1069 \\
\hline 5 & 1222 \\
\hline 6 & 1353 \\
\hline 7 & 1427 \\
\hline 8 & 1711 \\
\hline
\end{tabular}


Table 2. Estimated Lower detection limit (LDL) in ppm of acetone and the correlation coefficient (r) corresponding to eight peak wavenumbers for experiment 1,2,3 and 4 under condition-1(Three-replication three separate cuvette)

\begin{tabular}{|c|c|c|c|c|c|c|c|c|}
\hline $\begin{array}{c}\text { Peak } \\
\text { wavenumber } \\
\left(\mathrm{cm}^{-1}\right)\end{array}$ & $\begin{array}{c}\text { Est } \\
\text { LDL } \\
\text { (ppm) } \\
\text { Expt-1 }\end{array}$ & $\begin{array}{c}\text { Corr } \\
\text { Coeff } \\
(r)\end{array}$ & $\begin{array}{c}\text { Est LDL } \\
\text { (ppm) } \\
\text { Expt-2 }\end{array}$ & $\begin{array}{c}\text { Corr } \\
\text { Coeff (r) }\end{array}$ & $\begin{array}{c}\text { Est LDL } \\
\text { (ppm) } \\
\text { Expt-3 }\end{array}$ & $\begin{array}{c}\text { Corr } \\
\text { Coeff }(r)\end{array}$ & $\begin{array}{c}\text { Est } \\
\text { LDL } \\
\text { (ppm) } \\
\text { Expt-4 }\end{array}$ & $\begin{array}{c}\text { Corr } \\
\text { Coeff }(r)\end{array}$ \\
\hline 532 & 630.17 & 0.74 & 461.69 & 0.80 & 198.49 & 0.86 & 508.60 & 0.85 \\
\hline 766 & 592.71 & 0.55 & 356.53 & 0.85 & 204.99 & 0.84 & 443.56 & 0.88 \\
\hline 898 & 448.00 & 0.74 & 311.88 & 0.87 & 190.51 & 0.84 & 347.29 & 0.90 \\
\hline 1069 & 387.48 & 0.76 & 296.83 & 0.87 & 189.25 & 0.82 & 311.44 & 0.91 \\
\hline 1222 & 330.60 & 0.77 & 274.31 & 0.90 & 201.58 & 0.76 & 258.29 & 0.91 \\
\hline 1353 & 340.26 & 0.75 & 415.27 & 0.89 & 238.79 & 0.71 & 268.51 & 0.89 \\
\hline 1427 & 336.69 & 0.75 & 278.06 & 0.91 & 216.29 & 0.75 & 258.30 & 0.90 \\
\hline 1711 & 254.11 & 0.72 & 198.04 & 0.88 & 319.52 & 0.75 & 69.63 & 0.85 \\
\hline
\end{tabular}

Table 3. Estimated Lower detection limit (LDL) in ppm of acetone and the correlation coefficient (r) corresponding to eight peak wavenumbers for experiment 1, 2, 3 and 4 under condition-2. (Three-replication single cuvette)

\begin{tabular}{|c|c|c|c|c|c|c|c|c|}
\hline $\begin{array}{c}\text { Peak } \\
\text { wavenumber } \\
\left(\mathbf{c m}^{-1}\right)\end{array}$ & $\begin{array}{c}\text { Est LDL } \\
(\mathbf{p p m}) \\
\mathbf{E x p t - 1}\end{array}$ & $\begin{array}{c}\text { Corr } \\
\text { Coeff } \\
\mathbf{( r )}\end{array}$ & $\begin{array}{c}\text { Est LDL } \\
\mathbf{( p p m )} \\
\mathbf{E x p t - 2}\end{array}$ & $\begin{array}{c}\text { Corr } \\
\text { Coeff } \\
(\mathbf{r})\end{array}$ & $\begin{array}{c}\text { Est LDL } \\
(\mathbf{p p m}) \\
\mathbf{E x p t - 3}\end{array}$ & $\begin{array}{c}\text { Corr } \\
\text { Coeff } \\
(\mathbf{r})\end{array}$ & $\begin{array}{c}\text { Est LDL } \\
(\mathbf{p p m}) \\
\mathbf{E x p t - 4}\end{array}$ & $\begin{array}{c}\text { Corr } \\
\text { Coeff } \\
(\mathbf{r})\end{array}$ \\
\hline 532 & $\mathbf{4 3 . 4 5}$ & 0.92 & 35.5 & 0.95 & 27.06 & 0.95 & 26.89 & 0.96 \\
\hline 766 & 40.79 & 0.92 & 32.91 & 0.95 & 24.15 & 0.94 & 24.03 & 0.95 \\
\hline $\mathbf{8 9 8}$ & $\mathbf{2 9 . 0 5}$ & 0.93 & $\mathbf{2 7 . 9 5}$ & 0.94 & 13.76 & 0.93 & $\mathbf{1 7 . 9}$ & 0.94 \\
\hline 1069 & 32.33 & 0.92 & 34.26 & 0.94 & 13.5 & 0.93 & 22.22 & 0.94 \\
\hline 1222 & 33.54 & 0.93 & 29.62 & 0.95 & $\mathbf{9 . 7 8}$ & 0.93 & 19.18 & 0.95 \\
\hline 1353 & 31.34 & 0.93 & 29.78 & 0.95 & 12.47 & 0.93 & 18.35 & 0.95 \\
\hline 1427 & 30.33 & 0.93 & 30.37 & 0.94 & 12.6 & 0.94 & 18.53 & 0.95 \\
\hline 1711 & 32.66 & 0.93 & 30.55 & 0.95 & 20 & 0.94 & 19.26 & 0.95 \\
\hline
\end{tabular}




\section{Condition -1 (4 experiments)}

Three replications of each concentration

Three separate cuvettes for three replication

\section{Condition -2 (4 experiments)}

Three replications of each concentration

Single cuvette for three replication

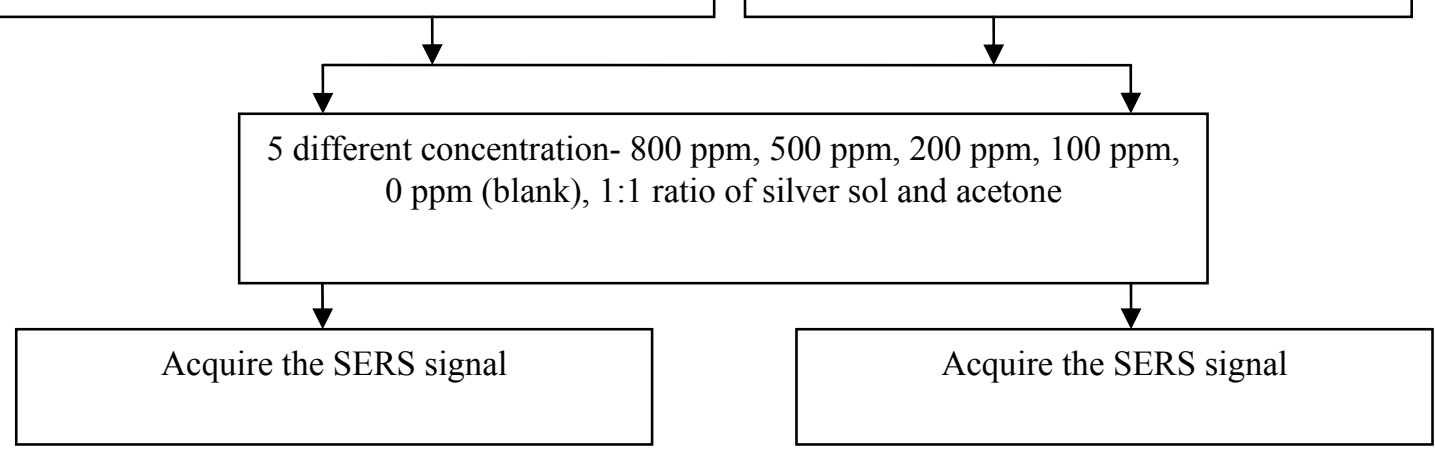

Figure 1. Experimental design for condition-1 and condition-2 of experiments

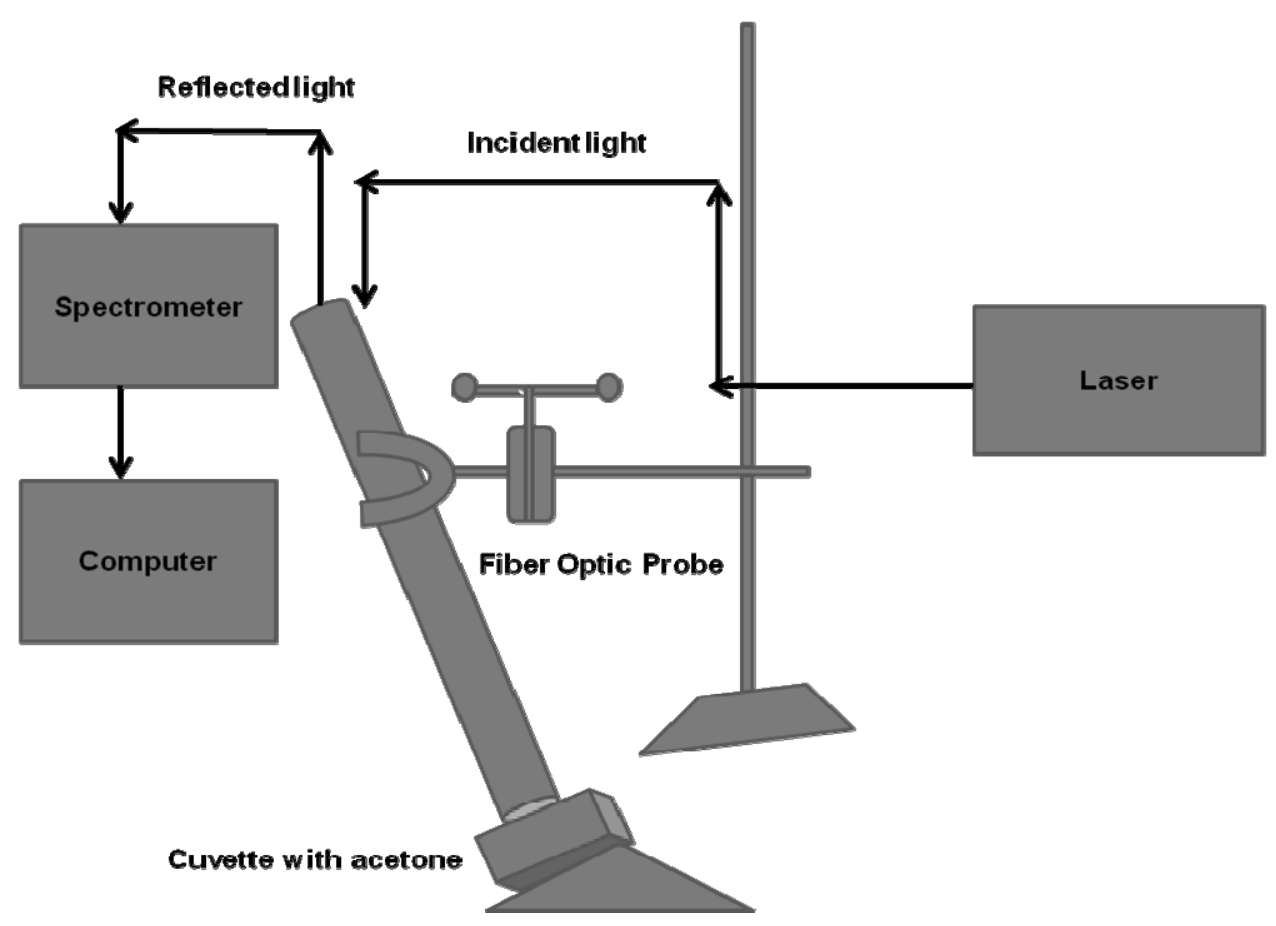

Figure 2. Schematic diagram of the SERS unit 


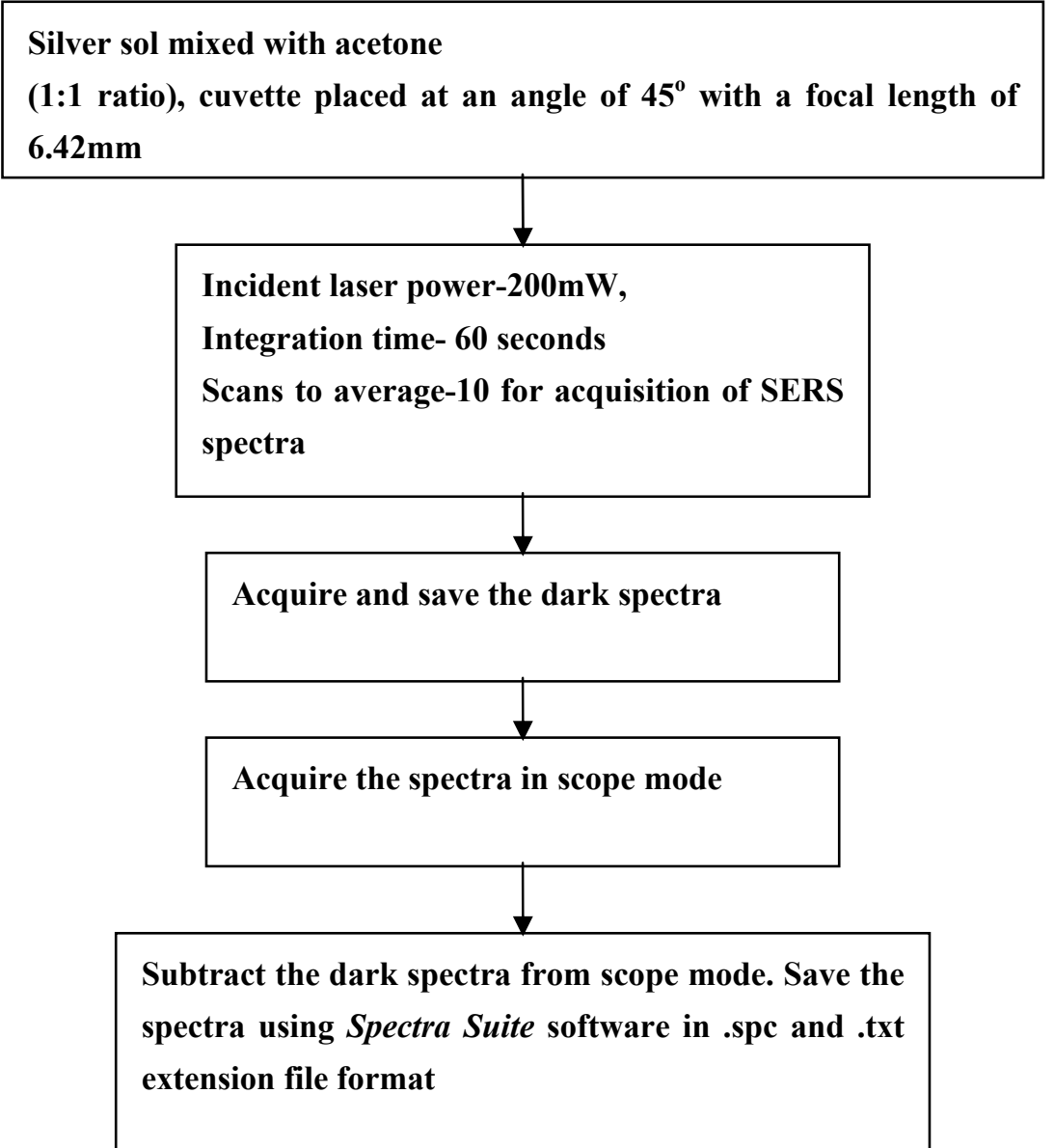

Figure 3. Flow chart for acquisition of Surface Enhanced Raman spectroscopy spectra using Spectra Suite software

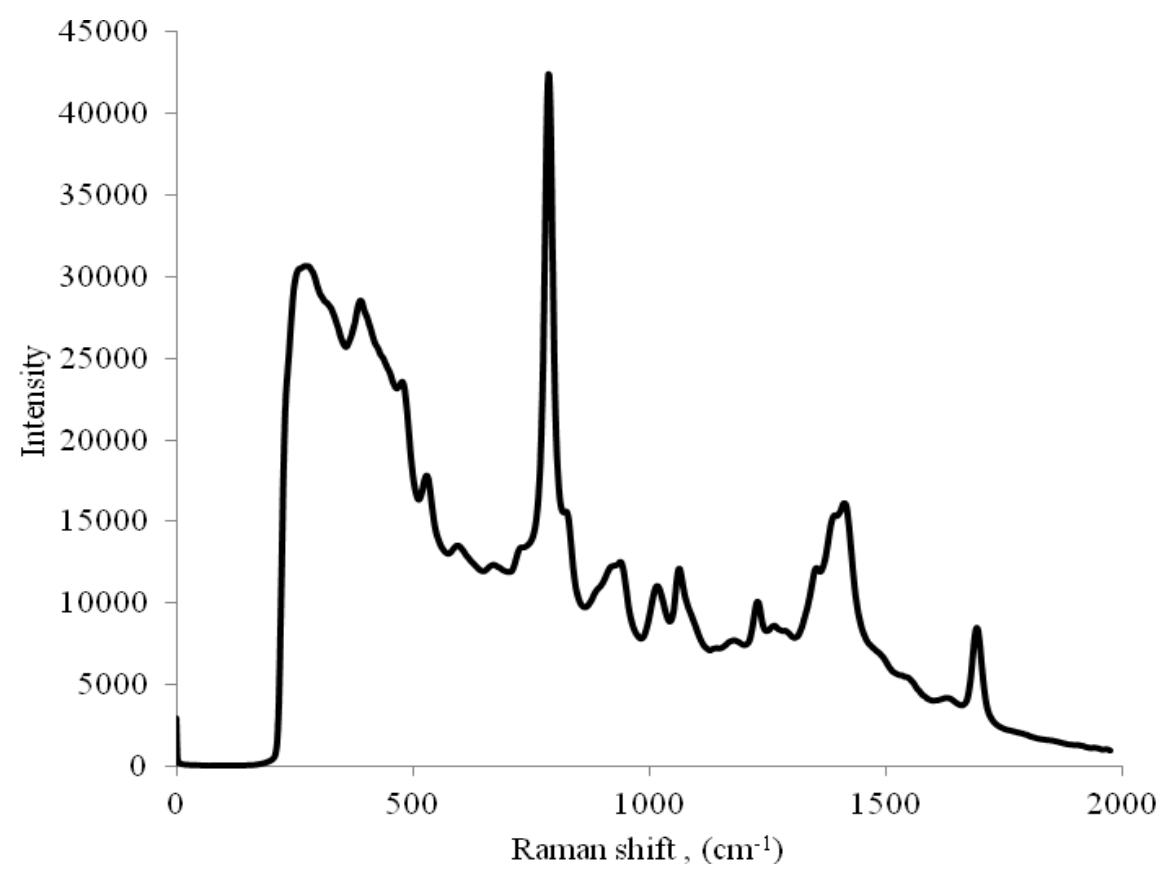

Figure 4. Spectral signature of acetone obtained using silver sol as SERS substrate 


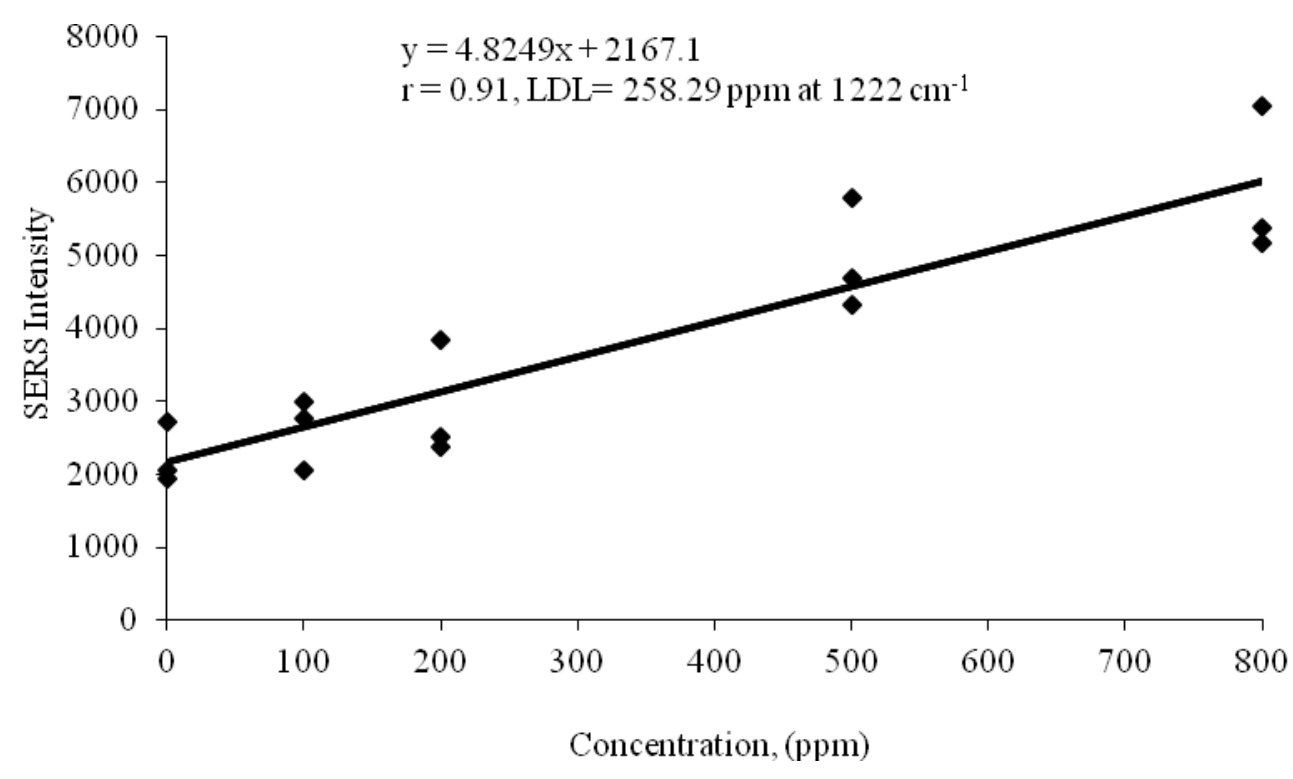

Figure 5. Estimated LDL of acetone at $1222 \mathrm{~cm}^{-1}$ for experiment 4 under condition-1

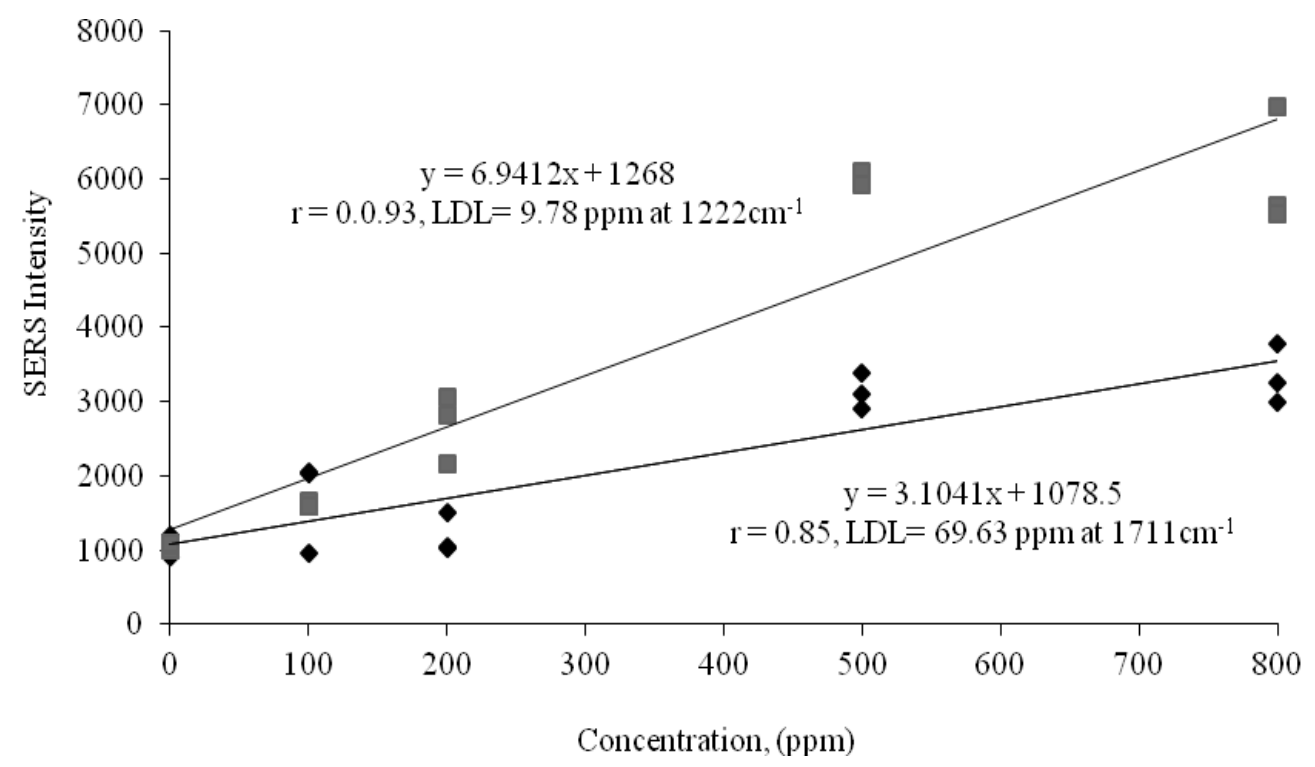

Figure 6. Lowest estimated LDL of acetone at $1711 \mathrm{~cm}^{-1}$ and $1222 \mathrm{~cm}^{-1}$ for under condition- 1 and 2 respectively 